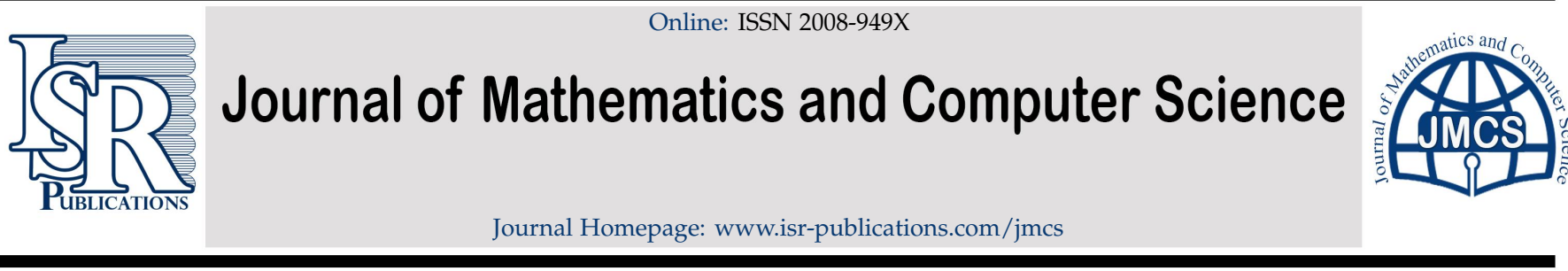

\title{
How to obtain Lie point symmetries of PDEs
}

\author{
Sajid Mohammad Kadhim, Mayada Gassab Mohammad, Hassan Kamil Jassim* \\ Departement of Mathematics, College Of Education for Pure Science, University Of Thi-Qar, Nasiriyah, Iraq.
}

\begin{abstract}
In this research, we have studied how to obtain Lie point symmetries of a partial differential equation (PDEs) of second order. We have also studied some PDEs' applications as one-dimensional and two-dimensional heat equations. We have used Manale's formula for solving second-order ordinary differential equations to determine new symmetries. Burgers equation has been studied, and Lie point symmetries have been obtained for these equations.
\end{abstract}

Keywords: Symmetries, infinitesimal, invariant condition.

2020 MSC: $34 \mathrm{C} 14,34 \mathrm{~A} 26$.

(C)2021 All rights reserved.

\section{Introduction}

More than a century ago, the Norwegian mathematician Sophus Lie put forward many of the fundamental ideas behind symmetry methods. The Lie symmetry method is a modern approximated method of solving of DEs and The Lie symmetry method (LSM) is efficient technique to find the approximate solution for ODEs and PDEs which describe different fields or science, physical phenomena, engineering,mechanics and so on. Sophus Lie (1872): develop DE invariant of continuous groups transformation. Hermann (1928) coined the term "Lie group", Bluman and Kumei (1989): dealt with symmetries and DEs, Hydone (2005): solved some DEs using Lie symmetry, Laheeb Muhsen (2015): develop the Lie symmetry analysis, especially Lie group analysis method to classify higher-order DDEs to solvable Lie algebra. So Lie symmetry method is necessary to obtain exact solutions or numerical solutions for partial differential equations (PDEs). During last few decades several analytical numerical and semi-analytical methods have been used for solving PDEs [4-6, 14-18]. In this paper we consider the symmetry analysis as presented in the books of Bluman, Kumei, Ibragimov and Anco [8, 9, 13]. We present the Lie symmetries of first-order PDEs and we find the Lie symmetry of Burgers equation [12] and finally we find the Lie symmetries of one-dimensional and two-dimensional heat equations.

\footnotetext{
*Corresponding author

Email address: hassan. kamil@yahoo.com (Hassan Kamil Jassim)

doi: $10.22436 /$ jmcs.022.04.01
} 


\section{Scalar PDEs with two dependent variables}

Definition 2.1 (Point symmetry of PDE [12]). Consider PDEs with one dependent variable $u$ and two independent variables $x$ and $t$. A point transformation is called diffeomorphism

$$
X=(x, t, u) \mapsto(\hat{x}(x, t, u), \hat{t}(x, t, u), \hat{u}(x, t, u)),
$$

this transformation maps the surface $u=u(x, t)$ to the following surface

$$
\hat{x}=\hat{x}(x, t, u(x, t)), \quad \hat{t}=\hat{t}(x, t, u(x, t)), \quad \hat{u}=\hat{u}(x, t, u(x, t)),
$$

to calculate the extend of the generator of a given transformation, we should find the derivatives of (2.1) with respect to each $x$ and $t$. We introduce the following total derivatives

$$
D_{x}=\partial_{x}+u_{x} \partial_{\mathfrak{u}}+u_{x x} \partial_{u_{x}}+u_{x t} \partial_{u_{t}}+\cdots, \quad D_{t}=\partial_{t}+u_{t} \partial_{\mathfrak{u}}+u_{x t} \partial_{u_{x}}+u_{t t} \partial_{u_{t}}+\cdots .
$$

Now if the jacobian

$$
\mathbf{J} \equiv\left|\begin{array}{ll}
D_{x} \hat{x} & D_{x} \hat{t} \\
D_{t} \hat{x} & D_{t} \hat{t}
\end{array}\right| \neq 0, \quad \text { when } \quad u=u(x, t)
$$

then the first two equations of (2.1) may be inverted to give $x$ and $t$ in terms of $\hat{x}$ and $\hat{t}$. If Eq. (2.2) holds then the last equation of (2.1) becomes

$$
\hat{u}=\hat{u}(\hat{x}, \hat{t}),
$$

now by using chain rule to (2.3), we get

$$
\left[\begin{array}{l}
D_{x} \hat{u} \\
D_{t} \hat{u}
\end{array}\right]=\left[\begin{array}{ll}
D_{x} \hat{x} & D_{x} \hat{t} \\
D_{t} \hat{x} & D_{t} \hat{t}
\end{array}\right]\left[\begin{array}{l}
\hat{u}_{\hat{x}} \\
\hat{u}_{\hat{t}}
\end{array}\right]
$$

now by Cramer's rule we find the transformation of the first derivatives

$$
\hat{u}_{\hat{x}}=\frac{1}{\mathbf{J}}\left|\begin{array}{ll}
D_{x} \hat{u} & D_{x} \hat{t} \\
D_{t} \hat{u} & D_{t} \hat{t}
\end{array}\right|, \quad \hat{u}_{\hat{t}}=\frac{1}{J}\left|\begin{array}{cc}
D_{x} \hat{x} & D_{x} \hat{u} \\
D_{t} \hat{x} & D_{t} \hat{u}
\end{array}\right|,
$$

now if $\hat{u}_{h}$ any derivative of $\hat{u}$ with respect to $\hat{x}$ and $\hat{t}$, then

$$
\hat{u}_{h \hat{x}} \equiv \frac{\partial \hat{u}_{h}}{\partial \hat{x}}=\frac{1}{J}\left|\begin{array}{ll}
D_{x} \hat{u}_{h} & D_{x} \hat{t} \\
D_{t} \hat{u}_{h} & D_{t} \hat{t}
\end{array}\right|, \quad \hat{u}_{h \hat{t}} \equiv \frac{\partial \hat{u}_{h}}{\partial \hat{t}}=\frac{1}{J}\left|\begin{array}{ll}
D_{x} \hat{x} & D_{x} \hat{u}_{h} \\
D_{t} \hat{x} & D_{t} \hat{u}_{h}
\end{array}\right| .
$$

Now we find the transformation of second derivatives

$$
\hat{u}_{\hat{x} \hat{x}}=\frac{1}{J}\left|\begin{array}{ll}
D_{x} \hat{u}_{\hat{x}} & D_{x} \hat{t} \\
D_{t} \hat{u}_{\hat{x}} & D_{t} \hat{t}
\end{array}\right|, \hat{u}_{\hat{t} \hat{t}}=\frac{1}{J}\left|\begin{array}{ll}
D_{x} \hat{x} & D_{x} \hat{u}_{\hat{t}} \\
D_{t} \hat{x} & D_{t} \hat{u}_{\hat{t}}
\end{array}\right|, \hat{u}_{\hat{x} \hat{t}}=\frac{1}{J}\left|\begin{array}{ll}
D_{x} \hat{u}_{\hat{t}} & D_{x} \hat{t} \\
D_{t} \hat{u}_{\hat{t}} & D_{t} \hat{t}
\end{array}\right|=\frac{1}{J}\left|\begin{array}{ll}
D_{x} \hat{x} & D_{x} \hat{u}_{\hat{x}} \\
D_{t} \hat{x} & D_{t} \hat{u}_{\hat{x}}
\end{array}\right| .
$$

Since

$$
\hat{u}_{\hat{t}}+\hat{u} \hat{u}_{\hat{x}}=8 t^{3}\left(u_{t}+u u_{x}\right),
$$

so the point transformation holds the symmetry condition

$$
\hat{u}_{\hat{t}}+\hat{u} \hat{u}_{\hat{x}}=\hat{u}_{\hat{x} \hat{x}} \quad \text { when } \quad u_{t}+u u_{x}=u_{x x} .
$$

Definition 2.2 (Point symmetries Of $n^{\text {th }}$ order PDE [12]). Consider

$$
f\left(x, t, u, u_{x}, u_{t}, \ldots\right)=0,
$$

the point transformation $X$ is a point symmetry of (2.6) if

$$
f\left(\hat{x}, \hat{t}, \hat{u}, \hat{u}_{\hat{x}}, \hat{u}_{\hat{t}}, \ldots\right)=0, \quad \text { when (2.6) holds. }
$$


We seek point symmetries of the form

$$
\hat{x}=x+\lambda \epsilon(x, t, u)+O\left(\lambda^{2}\right), \quad \hat{t}=t+\lambda \mu(x, t, u)+O\left(\lambda^{2}\right), \quad \hat{u}=u+\lambda \zeta(x, t, u)+O\left(\lambda^{2}\right) .
$$

In analogy with ODEs we define $\hat{u}_{\hat{x}}$ and $\hat{u}_{\hat{t}}$ as

$$
\hat{u}_{\hat{x}}=u_{x}+\lambda \zeta^{(x)}\left(x, t, u, u_{x}, u_{t}\right)+O\left(\lambda^{2}\right), \quad \hat{u}_{\hat{t}}=u_{t}+\lambda \zeta^{(t)}\left(x, t, u, u_{x}, u_{t}\right)+O\left(\lambda^{2}\right) .
$$

Now by using (2.4) we find $\hat{u}_{t}$ and $\hat{u}_{x}$ as follows

$$
\hat{u}_{t}=\frac{\left|\begin{array}{cc}
D_{x} \hat{x} & D_{x} \hat{u} \\
D_{t} \hat{x} & D_{t} \hat{u}
\end{array}\right|}{\left|\begin{array}{cc}
D_{x} \hat{x} & D_{x} \hat{t} \\
D_{t} \hat{x} & D_{t} \hat{t}
\end{array}\right|}=\frac{\left|\begin{array}{cc}
1+\lambda D_{x}(\epsilon)+O\left(\lambda^{2}\right) & u_{x}+\lambda D_{x}(\tau)+O\left(\lambda^{2}\right) \\
\lambda D_{t}(\epsilon)+O\left(\lambda^{2}\right) & u_{t}+\lambda D_{t}(\tau)+O\left(\lambda^{2}\right)
\end{array}\right|}{\left|\begin{array}{cc}
1+\lambda D_{x}(\epsilon)+O\left(\lambda^{2}\right) & \lambda D_{x}(\mu)+O\left(\lambda^{2}\right) \\
\lambda D_{t}(\epsilon)+O\left(\lambda^{2}\right) & 1+\lambda D_{t}(\mu)+O\left(\lambda^{2}\right)
\end{array}\right|}
$$

we ignore terms of $\left(\lambda^{2}\right)$ or higher, we arrive to

$$
\hat{u}_{t}=\frac{u_{t}+\lambda D_{t}(\zeta)+\lambda u_{t} D_{x}(\epsilon)-\lambda u_{x} D_{t}(\epsilon)+O\left(\lambda^{2}\right)}{1+\lambda D_{t}(\mu)+\lambda D_{X}(\epsilon)+O\left(\lambda^{2}\right)}=\frac{u_{t}+\lambda\left[D_{t}(\zeta)+u_{t} D_{x}(\epsilon)-u_{x} D_{t}(\epsilon)\right]+O\left(\lambda^{2}\right)}{1+\lambda\left[D_{t}(\mu)+D_{x}(\epsilon)\right]+O\left(\lambda^{2}\right)},
$$

since

$$
\hat{u}_{\hat{t}}=u_{t}+\lambda \zeta^{(t)}\left(x, t, u, u_{x}, u_{t}\right)+O\left(\lambda^{2}\right)
$$

then

$$
u_{t}+\lambda \zeta^{(t)}\left(x, t, u, u_{x}, u_{t}\right)+O\left(\lambda^{2}\right)=\frac{u_{t}+\lambda\left[D_{t}(\zeta)+u_{t} D_{x}(\epsilon)-u_{x} D_{t}(\epsilon)\right]+O\left(\lambda^{2}\right)}{1+\lambda\left[D_{t}(\mu)+D_{x}(\epsilon)\right]+O\left(\lambda^{2}\right)}
$$

this leads to

$$
\begin{aligned}
u_{t}+\lambda\left[D_{t}(\zeta)+u_{t} D_{x}(\epsilon)-u_{x} D_{t}(\epsilon)\right]+O\left(\lambda^{2}\right) & =\left(1+\lambda\left[D_{t}(\mu)+D_{x}(\epsilon)\right]+O\left(\lambda^{2}\right)\right)\left(u_{t}+\lambda \zeta^{(t)}\right) \\
& =u_{t}+u_{t} \lambda D_{t}(\mu)+u_{t} \lambda D_{x}(\epsilon)+\lambda \zeta^{(t)}+O\left(\lambda^{2}\right)
\end{aligned}
$$

by simplifying the Eq. (2.7) more yields

$$
\lambda\left[D_{t}(\zeta)+u_{t} D_{x}(\epsilon)-u_{x} D_{t}(\epsilon)-u_{t} D_{t}(\mu)-u_{t} D_{x}(\epsilon)\right]=\lambda \zeta^{(t)},
$$

so we obtain

$$
\zeta^{(t)}\left(x, t, u, u_{x}, u_{t}\right)=D_{t}(\zeta)-u_{x} D_{t}(\epsilon)-u_{t} D_{t}(\mu)
$$

In the same way we obtain

$$
\zeta^{(x)}\left(x, t, u, u_{x}, u_{t}\right)=D_{x}(\zeta)-u_{x} D_{x}(\epsilon)-u_{t} D_{x}(\mu),
$$

then

$$
\begin{aligned}
\zeta^{(t)} & =\left(\frac{\partial \zeta}{\partial t}+\frac{d u}{d t} \frac{\partial \zeta}{\partial u}\right)-u_{x}\left(\frac{\partial \epsilon}{\partial t}+\frac{d u}{d t} \frac{\partial \epsilon}{\partial u}\right)-u_{t}\left(\frac{\partial \mu}{\partial t}+\frac{d u}{d t} \frac{\partial \mu}{\partial u}\right) \\
& =\left(\zeta_{t}+u_{t} \zeta_{u}\right)-u_{x}\left(\epsilon_{t}+u_{t} \epsilon_{u}\right)-u_{t}\left(\mu_{t}+u_{t} \mu_{u}\right)=\zeta_{t}-\epsilon_{t} u_{x}+\left(\zeta_{u}-\mu_{t}\right) u_{t}-\epsilon_{u} u_{x} u_{t}-\mu_{u}\left(u_{t}^{2}\right),
\end{aligned}
$$

so

$$
\zeta^{(t)}=\zeta_{t}-\epsilon_{t} u_{x}+\left(\zeta_{\mathfrak{u}}-\mu_{t}\right) u_{t}-\epsilon_{\mathfrak{u}} u_{x} u_{t}-\mu_{u}\left(u_{t}^{2}\right),
$$

and

$$
\zeta^{(x)}=\left(\frac{\partial \zeta}{\partial x}+\frac{d u}{d x} \frac{\partial \zeta}{\partial u}\right)-u_{x}\left(\frac{\partial \epsilon}{\partial x}+\frac{d u}{d x} \frac{\partial \epsilon}{\partial u}\right)-u_{t}\left(\frac{\partial \mu}{\partial x}+\frac{d u}{d x} \frac{\partial \mu}{\partial u}\right)
$$




$$
\begin{aligned}
& =\left(\zeta_{x}+u_{x} \zeta_{\mathfrak{u}}\right)-u_{x}\left(\epsilon_{x}+u_{x} \epsilon_{\mathfrak{u}}\right)-u_{t}\left(\mu_{x}+u_{x} \mu_{\mathfrak{u}}\right) \\
& =\zeta_{x}+\left(\zeta_{\mathfrak{u}}-\epsilon_{x}\right) u_{x}-\mu_{x} u_{t}-\epsilon_{\mathfrak{u}}\left(u_{x}^{2}\right)-\mu_{\mathfrak{u}} u_{x} u_{\mathfrak{t}}
\end{aligned}
$$

so

$$
\zeta^{(x)}=\zeta_{x}+\left(\zeta_{\mathfrak{u}}-\epsilon_{x}\right) u_{x}-\mu_{x} u_{t}-\epsilon_{u}\left(u_{x}^{2}\right)-\mu_{u} u_{x} u_{t} .
$$

Now by using (2.5) the transformation is extend to higher-order derivatives frequently, assume that

$$
\hat{u}_{h}=u_{h}+\lambda \zeta^{(h)}+O\left(\lambda^{2}\right)
$$

where

$$
u_{h} \equiv \frac{\partial^{h_{1}+h_{2}} u}{\partial x^{h_{1}} \partial t^{h_{2}}}, \quad \hat{u}_{h} \equiv \frac{\partial^{h_{1}+h_{2}} \hat{u}}{\partial \hat{x}^{h_{1}} \partial \hat{t}^{h_{2}}}
$$

for some $h_{1}$ and $h_{2}$, then

$$
\hat{u}_{h \hat{x}}=u_{h x}+\lambda \zeta^{(h x)}+O\left(\lambda^{2}\right), \quad \hat{u}_{h \hat{t}}=u_{h t}+\lambda \zeta^{(h t)}+O\left(\lambda^{2}\right),
$$

from Eq. (2.5)

$$
\begin{aligned}
& \hat{u}_{h \hat{x}}=\frac{\left|\begin{array}{cc}
D_{x} \hat{u}_{h} & D_{x} \hat{t} \\
D_{t} \hat{u}_{h} & D_{t} \hat{t}
\end{array}\right|}{\left|\begin{array}{ll}
D_{x} \hat{x} & D_{x} \hat{t} \\
D_{t} \hat{x} & D_{t} \hat{t}
\end{array}\right|}=\frac{\left|\begin{array}{cc}
u_{h x}+\lambda D_{x}\left(\zeta^{(h)}\right)+O\left(\lambda^{2}\right) & \lambda D_{x}(\mu)+O\left(\lambda^{2}\right) \\
u_{h t}+\lambda D_{t}\left(\zeta^{(h)}\right)+O\left(\lambda^{2}\right) & 1+\lambda D_{t}(\mu)+O\left(\lambda^{2}\right)
\end{array}\right|}{\left|\begin{array}{cc}
1+\lambda D_{x}(\epsilon)+O\left(\lambda^{2}\right) & \lambda D_{x}(\mu)+O\left(\lambda^{2}\right) \\
\lambda D_{t}(\epsilon)+O\left(\lambda^{2}\right) & 1+\lambda D_{t}(\mu)+O\left(\lambda^{2}\right)
\end{array}\right|} \\
&=\frac{u_{h x}+\lambda u_{h x} D_{t}(\mu)-\lambda u_{h t} D_{x}(\mu)+O\left(\lambda^{2}\right)}{1+\lambda\left(D_{t}(\mu)+D_{x}(\epsilon)\right)+O\left(\lambda^{2}\right)},
\end{aligned}
$$

since

$$
\hat{u}_{h \hat{x}}=u_{h x}+\lambda \zeta^{(h x)}+O\left(\lambda^{2}\right),
$$

then

$$
\begin{aligned}
u_{h x}+\lambda u_{h x} D_{t}(\mu)+\lambda D_{x}\left(\zeta^{(h)}\right)-\lambda u_{h t} D_{x}(\mu)+O\left(\lambda^{2}\right) & =\left(1+\lambda\left(D_{t}(\mu)+D_{x}(\epsilon)\right)\right)\left(u_{h x}+\lambda \zeta^{(h x)}+O\left(\lambda^{2}\right)\right) \\
& =u_{h x}+\lambda u_{h x} D_{t}(\mu)+\lambda u_{h x} D_{x}(\epsilon)+\lambda \zeta^{(h x)}+O\left(\lambda^{2}\right),
\end{aligned}
$$

then

$$
u_{h x}+\lambda u_{h x} D_{t}(\mu)+\lambda D_{x}\left(\zeta^{(h)}\right)-\lambda u_{h t} D_{x}(\mu)-u_{h x}-\lambda u_{h x} D_{t}(\mu)-\lambda u_{h x} D_{x}(\epsilon)=\lambda \zeta^{(h x)},
$$

then

$$
\lambda\left(D_{\chi}\left(\zeta^{h}\right)-u_{h x} D_{\chi}(\epsilon)-u_{h t} D_{\chi}(\mu)\right)=\lambda \zeta^{(h x)},
$$

so

$$
\zeta^{(h x)}=D_{x}\left(\zeta^{h}\right)-u_{h x} D_{x}(\epsilon)-u_{h t} D_{x}(\mu) .
$$

In the same way

$$
\zeta^{(h t)}=D_{t}\left(\zeta^{h}\right)-u_{h x} D_{t}(\epsilon)-u_{h t} D_{t}(\mu) .
$$

We introduce the infinitesimal generator $X$

$$
X=\epsilon \partial_{x}+\mu \partial_{t}+\zeta \partial_{u} .
$$


The infinitesimal generator is extend to derivatives by adding all terms of the form $\zeta^{(h)} \partial_{\mathfrak{u}_{h}}$ up to the desired order, the first extension as

$$
X^{[1]}=X+\zeta^{(x)} \partial_{u_{x}}+\zeta^{(t)} \partial_{u_{t}}
$$

is a point symmetry of $f\left(x, t, u, u_{x}, u_{t}, \ldots\right)=0$ if Lie's symmetry condition

$$
\left.X^{[1]} f\right|_{f=0}
$$

and the second extension $X^{[2]}$ of the operator $X$ is

$$
X^{[2]}=X^{[1]}+\zeta^{(x x)} \partial_{u_{x x}}+\zeta^{(x t)} \partial_{u_{x t}}+\zeta^{t t} \partial_{u_{\mathfrak{t t}}}
$$

is a point symmetry of $f\left(x, t, u, u_{x}, u_{t}, \ldots\right)=0$ if Lie's symmetry condition $\left.X^{[2]} f\right|_{f=0}$ and the extended transformations are given as

$$
\begin{aligned}
& \zeta^{(x x)}=D_{x}\left(\zeta^{(x)}\right)-u_{x x} D_{x}(\epsilon)-u_{x t} D_{x}(\mu), \\
& \zeta^{(x t)}=D_{t}\left(\zeta^{(x)}\right)-u_{x x} D_{t}(\epsilon)-u_{x t} D_{t}(\mu),
\end{aligned}
$$

and

$$
\begin{aligned}
& \zeta^{(x t)}=D_{x}\left(\zeta^{(t)}\right)-u_{x t} D_{x}(\epsilon)-u_{t t} D_{x}(\mu), \\
& \zeta^{(t t)}=D_{t}\left(\zeta^{(t)}\right)-u_{x t} D_{t}(\epsilon)-u_{t t} D_{t}(\mu),
\end{aligned}
$$

now we expand $\left.\left.\zeta^{(x x)}\right), \zeta^{(x t)}\right)$ and $\left.\zeta^{(t t)}\right)$, we get

$$
\begin{aligned}
& \zeta^{(x x)}=\zeta_{x x}+\left(2 \zeta_{x u}-\epsilon_{x x}\right) u_{x}-\mu_{x x} u_{t}+\left(\zeta_{u u}-2 \epsilon_{x u}\right) u_{x}^{2} \\
& -2 \mu_{x u} u_{x} u_{t}-\epsilon_{u u} u_{x}^{3}-\mu_{u u} u_{x}^{2} u_{t}+\left(\zeta_{\mathfrak{u}}-2 \varepsilon_{x}\right) u_{x x} \\
& -2 \mu_{x} u_{x t}-3 \epsilon_{\mathfrak{u}} u_{x} u_{x x}-\mu_{\mathfrak{u}} u_{t} u_{x x}-2 \mu_{\mathfrak{u}} u_{x} u_{x t} \text {, } \\
& \zeta^{(x t)}=\zeta_{x t}+\left(\zeta_{t u}-\epsilon_{x t}\right) u_{x}+\left(\zeta_{x u}-\mu_{x t}\right) u_{t}-\epsilon_{t u} u_{x}^{2} \\
& +\left(\zeta_{\mathfrak{u u}}-\epsilon_{x u}-\mu_{t u}\right) u_{x} u_{t}-\mu_{x u} u_{t}^{2}-\epsilon_{u u} u_{x}^{2} u_{t}-\epsilon_{u u} u_{x} u_{t}^{2} \\
& -\epsilon_{t} u_{x x}-\epsilon_{u} u_{t} u_{x x}+\left(\zeta_{\mathfrak{u}}-\epsilon_{x}-\mu_{t}\right) u_{x t}-2 \epsilon_{u} u_{x} u_{x t}-2 \epsilon_{\mathfrak{u}} u_{t} u_{x t}-\epsilon_{x} u_{t t}-\epsilon_{u} u_{x} u_{t t} \text {, } \\
& \zeta^{(t t)}=\zeta_{t t}-\epsilon_{t t} u_{x}+\left(2 \zeta_{t u}-\epsilon_{t t}\right) u_{t}-2 \epsilon_{t u} u_{x} u_{t} \\
& +\left(\zeta_{u u}-2 \mu_{t u}\right) u_{t}^{2}-\epsilon_{u u} u_{x} u_{t}^{2}-\mu_{u u} u_{t}^{3}-2 \epsilon_{t} u_{x t} \\
& -2 \epsilon_{\mathfrak{u}} u_{t} u_{x t}+\left(\zeta_{\mathfrak{u}}-2 \mu_{t}\right) u_{t t}-\epsilon_{\mathfrak{u}} u_{x} u_{t t}-3 \mu_{u} u_{t} u_{t t} .
\end{aligned}
$$

Definition 2.3 (Lie algebra, [20]). A Lie algebra $L$ is a vector space $V$ of operations $X=\xi_{i} \frac{\partial}{\partial x_{i}}$ over a field (real or complex) together with a binary operator [,] $: \mathrm{V} \times \mathrm{V} \longrightarrow \mathrm{V}$ with the following properties:

Anti-symmetry/Anti-commutativity/skew-symmetry

$$
\left[X_{1}, X_{2}\right]=-\left[X_{2}, X_{1}\right]
$$

for all $X_{1}, X_{2} \in L$.

Bilinearity

$$
\left[X_{1}, \alpha X_{2}+\beta X_{3}\right]=\alpha\left[X_{1}, X_{2}\right]+\beta\left[X_{1}, X_{3}\right] \text { and }\left[\alpha X_{1}+\beta X_{2}, X_{3}\right]=\alpha\left[X_{1}, X_{3}\right]+\beta\left[X_{2}, X_{3}\right],
$$

where $\alpha, \beta$ are constants, for all $X_{1}, X_{2}, X_{3} \in L$. 
Jacobi Identity

$$
\left[\left[X_{1}, X_{2}\right], X_{3}\right]+\left[\left[X_{2}, X_{3}\right], X_{1}\right]+\left[\left[X_{3}, X_{1}\right], X_{2}\right]=0,
$$

for all $X_{1}, X_{2}, X_{3} \in L$, where

$$
X_{i}=\xi_{i} \frac{\partial}{\partial x_{i}}, \quad i=1,2, \ldots, n .
$$

For example, $\left[X_{1}, X_{2}\right]=X_{1} X_{2}-X_{2} X_{1}$ is also an element of $L$, so [., .] is called the Lie bracket or commutator of L.

Definition 2.4 ([9]). Let $X_{i}=\xi_{s} \frac{\partial}{\partial x_{s}}$ and $X_{j}=\eta_{s} \frac{\partial}{\partial x_{s}}, i, j=1, \ldots, r$ and $s=1, \ldots, n$ be two infinitesimal generator. The commutator $\left[X_{i}, X_{j}\right]$ of $X_{i}$ and $X_{j}$ is the first order operator

$$
\left[X_{i}, X_{j}\right]=X_{i} X_{j}-X_{j} X_{i}=\sum_{s}^{n} \sum_{m}^{n}\left(\xi_{m} \frac{\partial \eta_{s}}{\partial x_{m}}-\eta_{m} \frac{\partial \xi_{s}}{\partial x_{m}}\right) \frac{\partial}{\partial x_{s}} .
$$

Definition 2.5 ([20]). Lie groups $G$ is a smooth manifold and a group such that the multiplication $\rho: \mathrm{G} \times \mathrm{G} \longrightarrow \mathrm{G}$ is smooth. The inversion $\sigma: \mathrm{G} \longrightarrow \mathrm{G}$ is also smooth.

Definition $2.6([11,20])$. A finite set of infinitesimal generator $X_{1}, X_{2}, \ldots, X_{r}$ is said to be a basis for the Lie algebra $L$ if $X_{i} \in L$ and

- $\mathrm{X}_{1}, \mathrm{X}_{2}, \ldots, \mathrm{X}_{\mathrm{r}}$ form a basis of the vector space;

- $\left[X_{i}, X_{j}\right]=c_{i j k} X_{k}$.

The coefficients $c_{i j k}$ are called the structure constant of the Lie algebra, $i, j, k=1,2, \ldots, r$.

Theorem 2.7 (Second fundamental theorem of Lie, [9]). Any two infinitesimal generators of an r-parameter Lie group, satisfy commutation relation of the form $\left[X_{i}, X_{j}\right]=c_{i j k} X_{k}$, where $i, j, k=1,2, \ldots, r$. Provided the infinitesimal generators span the Lie algebra associated with the Lie group, all real (or complex) linear combinations of the $X_{i}$ will also obey commutation relations and the Jacobi identity associated with the Lie algebra. That is, the given infinitesimal generators $\mathrm{X}_{1}, \mathrm{X}_{2}, \ldots, \mathrm{X}_{\mathrm{r}}$ form a basis for the Lie algebra.

Theorem 2.8 ([13]). A function $\mathrm{F}(\mathrm{x})$ is an invariant under the Lie group of transformation if and only if $\mathrm{XF}(\mathrm{x})=0$, where $\mathrm{X}$ is an infinitesimal generator.

\section{Lie symmetry first-order PDEs [12]}

Consider the PDE

$$
u_{t}=u_{x}^{2}
$$

we determine the symmetries of Eq. (3.1). Using Theorem 2.8

$$
\left.X^{[2]} f\right|_{f=0}=0,
$$

where

$$
f=u_{t}-u_{x}^{2}=0
$$

and $X^{[2]}$ is the second-order extended of

$$
X=\epsilon(x, t, u) \frac{\partial}{\partial x}+\mu(x, t, u) \frac{\partial}{\partial t}+\zeta(x, t, u) \frac{\partial}{\partial u},
$$

namely,

$$
X^{[2]}=\epsilon \frac{\partial}{\partial x}+\mu \frac{\partial}{\partial t}+\zeta \frac{\partial}{\partial u}+\zeta^{(x)} \frac{\partial}{\partial u_{x}}+\zeta^{(t)} \frac{\partial}{\partial u_{t}}+\zeta^{(x x)} \frac{\partial}{\partial u_{x x}}+\zeta^{(x t)} \frac{\partial}{\partial u_{x t}}+\zeta^{(t t)} \frac{\partial}{\partial u_{t t}} .
$$


Hence we solve

$$
\left.\left(\epsilon \frac{\partial}{\partial x}+\mu \frac{\partial}{\partial t}+\zeta \frac{\partial}{\partial u}+\zeta^{(x)} \frac{\partial}{\partial u_{x}}+\zeta^{(t)} \frac{\partial}{\partial u_{t}}+\zeta^{(x x)} \frac{\partial}{\partial u_{x x}}+\zeta^{(x t)} \frac{\partial}{\partial u_{x t}}+\zeta^{(t t)} \frac{\partial}{\partial u_{t t}}\right)\left(u_{t}-u_{x}^{2}\right)\right|_{u_{t}=u_{x}^{2}}=0,
$$

so we get

$$
\zeta^{(t)}=2 u_{x} \zeta^{(x)}
$$

we can expand the Eq. (3.2) by using the Eqs. (2.8) and (2.9)

$$
\zeta_{t}-\epsilon_{t} u_{x}+\left(\zeta_{u}-\mu_{t}\right) u_{t}-\epsilon_{u} u_{x} u_{t}-\mu_{u} u_{t}^{2}=2 u_{x}\left(\zeta_{x}+\left(\zeta_{u}-\epsilon_{x}\right) u_{x}-\mu_{x} u_{t}-\epsilon_{u} u_{x}^{2}-\mu_{u} u_{x} u_{t}\right),
$$

now replace $u_{x}^{2}$ by $u_{t}$ we get

$$
\zeta_{t}-\epsilon_{t} \mu_{x}+\left(\zeta_{\mathfrak{u}}-\mu_{t}\right) u_{x}^{2}-\epsilon_{\mathfrak{u}} u_{x} u_{x}^{2}-\mu_{u} u_{x}^{4}=2 u_{x}\left(\zeta_{x}+\left(\zeta_{\mathfrak{u}}-\epsilon_{x}\right) u_{x}-\mu_{x} u_{x}^{2}-\epsilon_{\mathfrak{u}} u_{x}^{2}-\mu_{u} u_{x} u_{x}^{2}\right),
$$

more simplification we get

$$
\zeta_{t}-\left(\epsilon_{t}+2 \zeta_{x}\right) u_{x}+\left(-\zeta_{\mathfrak{u}}-\mu_{t}+2 \epsilon_{x}\right) u_{x}^{2}+\left(\epsilon_{\mathfrak{u}}+2 \mu_{x}\right) u_{x}^{3}+\mu_{\mathfrak{u}} u_{x}^{4}=0,
$$

then for Eq. (3.3) to be satisfied requires setting the coefficient of $u_{x}$ to zero we are left with the system of determining equations

$$
\begin{aligned}
\zeta_{\mathrm{t}} & =0, \\
\epsilon_{\mathrm{t}}+2 \zeta_{\mathrm{x}} & =0, \\
\zeta_{\mathrm{u}}+\mu_{\mathrm{t}}-2 \epsilon_{\mathrm{x}} & =0, \\
\epsilon_{\mathfrak{u}}+2 \mu_{\mathrm{x}} & =0, \\
\mu_{\mathfrak{u}} & =0,
\end{aligned}
$$

we begin by solving Eq. (3.8) by integration both side we get

$$
\mu=\alpha(x, t)
$$

where $\alpha$ is an arbitrary function.

Now integrating equation (3.7) with respect to $u$ and substituting

$$
\mu_{x}=\alpha_{x}(x, t),
$$

we get

$$
\epsilon=-2 \alpha_{x} u+\beta(x, t),
$$

then

$$
\epsilon_{x}=-2 \alpha_{x \chi} u+\beta_{x}(x, t) .
$$

From equation (3.6)

$$
\zeta_{\mathfrak{u}}=2 \epsilon_{\mathrm{x}}-\mu_{\mathrm{t}},
$$

substituting Eq. (3.11) into Eq. (3.12) we get

$$
\zeta_{\mathfrak{u}}=-4 \alpha_{x x} u+2 \beta_{x}(x, t)-\alpha_{t}(x, t),
$$

now integrating Eq. (3.13) with respect to $u$ we get

$$
\zeta=-2 \alpha_{x x} u^{2}+\left(2 \beta_{x}-\alpha_{t}\right) u+\gamma(x, t),
$$

for some functions $\beta$ and $\gamma$. Since

$$
\epsilon_{\mathrm{t}}=-2 \alpha_{x \mathrm{t}} \mathrm{u}+\beta_{\mathrm{t}}(\mathrm{x}, \mathrm{t}),
$$




$$
\begin{aligned}
& \zeta_{x}=-2 \alpha_{x x x} u^{2}+\left(2 \beta_{x x}-\alpha_{x t}\right) u+\gamma_{x}(x, t), \\
& \zeta_{t}=-2 \alpha_{x x t} u^{2}+\left(2 \beta_{x t}-\alpha_{t t}\right) u+\gamma_{t}(x, t),
\end{aligned}
$$

now by substituting Eqs. (3.15), (3.16), and (3.17) into Eqs. (3.4) and (3.5) we get

$$
-2 \alpha_{x t} u+\beta_{t}(x, t)-4 \alpha_{x x x} u^{2}+2\left(2 \beta_{x x}-\alpha_{x t}\right) u+2 \gamma_{x}(x, t)=0,
$$

more simplifying we get

$$
-4 \alpha_{x x x} u^{2}+4\left(\beta_{x x}-\alpha_{x t}\right) u+\beta_{t}(x, t)+2 \gamma_{x}(x, t)=0 .
$$

Substituting Eq. (3.17) in to Eq. (3.4) we get

$$
-2 \alpha_{x x t} u^{2}+\left(2 \beta_{x t}-\alpha_{t t}\right) u+\gamma_{t}(x, t)=0,
$$

such that $\alpha, \beta$ and $\gamma$ are independent of $u$. Now splitting Eq. (3.18) and (3.19) with respect to the power of $u$ we get

$$
\begin{aligned}
\gamma_{t} & =0, \\
\beta_{x x}-\alpha_{x t} & =0, \\
2 \beta_{x t}-\alpha_{t t} & =0, \\
\beta_{t}+2 \gamma_{x} & =0, \\
\alpha_{x x x} & =0, \\
\alpha_{x x t} & =0,
\end{aligned}
$$

integrating Eq. (3.20) we get

$$
\gamma=\mathrm{B}_{1}(\mathrm{x})
$$

From equation (3.23)

$$
\beta_{t}(x, t)=-2 \gamma_{x}(x, t),
$$

then integrating equation (3.27) with respect to $t$ we get

$$
\beta(x, t)=-2 B_{1}^{\prime}(x) t+B_{2}(x) .
$$

From Eq. (3.22) we get

$$
\alpha_{\mathrm{tt}}=-4 \mathrm{~B}_{1}^{\prime \prime}(\mathrm{x})
$$

by integrating Eq. (3.29) twice with respect to $t$ we get

$$
\alpha=-2 B_{1}^{\prime \prime}(x) t^{2}+B_{3}(x) t+B_{4}(x),
$$

where $B_{1}, B_{2}, B_{3}$ and $B_{4}$ are function of $x$. From Eq. (3.26), (3.28), and (3.30) we can find

$$
\begin{array}{rlrl}
\alpha_{x x x} & =-2 B_{1}^{\prime \prime \prime \prime \prime}(x) t^{2}+B_{3}^{\prime \prime \prime}(x) t+B_{4}^{\prime \prime \prime}(x), \quad \alpha_{x x t} & =-4 B_{1}^{\prime \prime \prime \prime}(x) t+B_{3}^{\prime \prime}(x), \\
\beta_{x x} & =-2 B_{1}^{\prime \prime \prime}(x) t+B_{2}^{\prime \prime}(x), & \alpha_{x t} & =-4 B_{1}^{\prime \prime \prime}(x) t+B_{3}^{\prime}(x),
\end{array}
$$

now substituting Eq. (3.31) in to Eq. (3.21), (3.24), and (3.25) we get

$$
2 \mathrm{~B}_{1}^{\prime \prime} \mathrm{t}+\mathrm{B}_{2}^{\prime \prime}(\mathrm{x})-\mathrm{B}_{3}^{\prime}(\mathrm{x})=0, \quad-2 \mathrm{~B}_{1}^{\prime \prime \prime \prime \prime}(x) \mathrm{t}^{2}+\mathrm{B}_{3}^{\prime \prime \prime}(x) \mathrm{t}+\mathrm{B}_{4}^{\prime \prime \prime}(\mathrm{x})=0, \quad-4 \mathrm{~B}_{1}^{\prime \prime \prime \prime}(x) \mathrm{t}+\mathrm{B}_{3}^{\prime \prime}(\mathrm{x})=0,
$$

equating powers of $t$ and solving the resulting ODEs we get

$$
\begin{aligned}
\mathrm{B}_{1}^{\prime \prime \prime} & =0, \\
\mathrm{~B}_{2}^{\prime \prime}-\mathrm{B}_{3}^{\prime} & =0,
\end{aligned}
$$




$$
\begin{aligned}
\mathrm{B}_{3}^{\prime \prime} & =0, \\
\mathrm{~B}_{4}^{\prime \prime \prime} & =0,
\end{aligned}
$$

we begin by solving Eq. (3.32) to obtain

$$
\mathrm{B}_{1}(x)=\frac{1}{2} \mathrm{c}_{1} x^{2}+\mathrm{c}_{2} x+\mathrm{c}_{3}
$$

From Eqs. (3.33), (3.34), and (3.35) we obtain

$$
\begin{aligned}
& \mathrm{B}_{2}(x)=\frac{1}{2} c_{4} x^{2}+c_{6} x+c_{7} \\
& B_{3}(x)=c_{4} x+c_{5} \\
& B_{4}(x)=\frac{1}{2} c_{8} x^{2}+c_{9} x+c_{10} .
\end{aligned}
$$

Now we put $\beta_{1}=\frac{c_{1}}{2}, \beta_{2}=\frac{c_{2}}{2}, \beta_{4}=c_{4}, \beta_{5}=c_{5}, \beta_{6}=c_{6}, \beta_{7}=c_{7}, \beta_{8}=\frac{c_{8}}{2}, \beta_{9}=c_{9}$, and $\beta_{10}=c_{10}$, where $\beta_{1}, \ldots, \beta_{10}$ are arbitrary constants. Then the equations (3.36), (3.37), (3.38), and (3.39) become

$$
B_{1}(x)=\beta_{1} x^{2}+2 \beta_{2} x+\beta_{3}, \quad B_{2}(x)=\frac{1}{2} \beta_{4} x^{2}+\beta_{6} x+\beta_{7}, \quad B_{3}(x)=\beta_{4} x+\beta_{5}, \quad B_{4}(x)=\beta_{8} x^{2}+\beta_{9} x+\beta_{10} .
$$

So the Eqs. (3.9), (3.10), and (3.14) become

$$
\begin{aligned}
& \epsilon=-4 \beta_{1} x t-2 \beta_{2} t+\beta_{4}\left(\frac{1}{2} x^{2}-2 t u\right)+\beta_{6} x+\beta_{7}-4 \beta_{8} x u-2 \beta_{9} u, \\
& \mu=-4 \beta_{1} t^{2}+\beta_{4} x t+\beta_{5} t+\beta_{8} x^{2}+\beta_{9} x+\beta_{10}, \\
& \zeta=\beta_{1} x^{2}+\beta_{2} x+\beta_{3}+\beta_{4} x u-\beta_{5} u+2 \beta_{6} u-4 \beta_{8} u^{2} .
\end{aligned}
$$

Now we determine the symmetry of the Eq. (3.1). Since the generator

$$
X=\epsilon \frac{\partial}{\partial x}+\mu \frac{\partial}{\partial t}+\zeta \frac{\partial}{\partial u}
$$

substituting the infinitesimal (3.40) into Eq. (3.41) we get

$$
\begin{aligned}
X=- & 4 \beta_{1} x t \frac{\partial}{\partial x}-2 \beta_{2} t \frac{\partial}{\partial x}+\beta_{4}\left(\frac{1}{2} x^{2}-2 t u\right) \frac{\partial}{\partial x}+\beta_{6} x \frac{\partial}{\partial x}+\beta_{7} \frac{\partial}{\partial x}-4 \beta_{8} x u \frac{\partial}{\partial x}-2 \beta_{9} u \frac{\partial}{\partial x} \\
& -4 \beta_{1} t^{2} \frac{\partial}{\partial t}+\beta_{4} x t \frac{\partial}{\partial t}+\beta_{5} t \frac{\partial}{\partial t}+\beta_{8} x^{2} \frac{\partial}{\partial t}+\beta_{9} x \frac{\partial}{\partial t}+\beta_{10} \frac{\partial}{\partial t}+\beta_{1} x^{2} \frac{\partial}{\partial u}+\beta_{3} \frac{\partial}{\partial u} \\
& +\beta_{4} x u \frac{\partial}{\partial u}-\beta_{5} u \frac{\partial}{\partial u}+2 \beta_{6} u \frac{\partial}{\partial u}-4 \beta_{8} u^{2} \frac{\partial}{\partial u},
\end{aligned}
$$

By Theorem 2.8, the Lie algebra of Equation (3.1) is spanned by the following infinitesimal generators corresponding to each $\beta_{i}$

$$
\begin{aligned}
& X_{1}=\frac{\partial}{\partial u}, \quad X_{2}=\frac{\partial}{\partial x^{\prime}}, \quad X_{3}=\frac{\partial}{\partial t^{\prime}}, \\
& X_{4}=-4 x t \frac{\partial}{\partial x}-4 t^{2} \frac{\partial}{\partial t}+x^{2} \frac{\partial}{\partial u^{\prime}}, \\
& X_{5}=-2 t \frac{\partial}{\partial x}+x \frac{\partial}{\partial u^{\prime}}, \\
& X_{6}=\left(\frac{1}{2} x^{2}-2 t u\right) \frac{\partial}{\partial x}+x t \frac{\partial}{\partial t}+x u \frac{\partial}{\partial u}, \\
& X_{7}=t \frac{\partial}{\partial t}-u \frac{\partial}{\partial u},
\end{aligned}
$$




$$
\begin{aligned}
x_{8} & =x \frac{\partial}{\partial t}+2 u \frac{\partial}{\partial u}, \\
x_{9} & =-4 x u \frac{\partial}{\partial x}+x^{2} \frac{\partial}{\partial t}-4 u^{2} \frac{\partial}{\partial u} \\
x_{10} & =-2 u \frac{\partial}{\partial x}+x \frac{\partial}{\partial t} .
\end{aligned}
$$

\section{Lie symmetry of Burgers equation}

Burgers' equation is a fundamental partial differential equation occurring in various areas of applied mathematics, such as fluid mechanics, nonlinear acoustics, gas dynamics, traffic flow. The equation was first introduced by Harry Bateman in 1915 [7, 21] and later studied by Johannes Martinus Burgers in 1948 [10], therefore, it is necessary to find the Lie point symmetry of that equation.

In this section we will consider the one-dimensional Burgers equation to be the nonlinear parabolic PDE

$$
u_{t}+u u_{x}=u_{x x}
$$

now we determine the linearized symmetry condition for Eq. (4.1) then by using Lie's invariance condition is

$$
\left.X^{[2]} f\right|_{f=0}=0,
$$

where

$$
f=u_{t}+u u_{x}=u_{x x}
$$

and $X^{[2]}$ is the second-order extended of

$$
X=\epsilon(x, t, u) \frac{\partial}{\partial x}+\mu(x, t, u) \frac{\partial}{\partial t}+\zeta(x, t, u) \frac{\partial}{\partial u},
$$

namely

$$
X^{[2]}=\epsilon \frac{\partial}{\partial x}+\mu \frac{\partial}{\partial t}+\zeta \frac{\partial}{\partial u}+\zeta^{(x)} \frac{\partial}{\partial u_{x}}+\zeta^{(t)} \frac{\partial}{\partial u_{t}}+\zeta^{(x x)} \frac{\partial}{\partial u_{x x}}+\zeta^{(x t)} \frac{\partial}{\partial u_{x t}}+\zeta^{(t t)} \frac{\partial}{\partial u_{t t}},
$$

then the linearized symmetry condition for Eq. (4.1) is

$$
\zeta^{(t)}+u \zeta^{(x)}+u_{x} \zeta=\zeta^{(x x)} .
$$

Substituting Eq. (2.8), (2.9), and (2.10) into equation (4.2) we get

$$
\begin{aligned}
\zeta_{t}- & \epsilon_{t} u_{x}+\left(\zeta_{\mathfrak{u}}-\mu_{t}\right) u_{t}-\zeta_{\mathfrak{u}} u_{x} u_{t}-\mu_{\mathfrak{u}} u_{t}^{2}+u \zeta_{x}+u\left(\zeta_{\mathfrak{u}}-\epsilon_{x}\right) u_{x}-u \mu_{x} u_{t}-u \epsilon_{\mathfrak{u}} u_{x}^{2}-u \mu_{u} u_{t}^{2}+u_{x} \zeta \\
= & \zeta_{x x}+\left(2 \zeta_{x u}-\zeta_{x x}\right) u_{x}-\mu_{x x} u_{t} \\
& +\left(\zeta_{u u}-2 \zeta_{x u}\right) u_{x}^{2}-2 \mu_{x u} u_{x} u_{t}-\zeta_{u u} u_{x}^{3}-\mu_{u u} u_{x}^{2} u_{t}+\left(\zeta_{\mathfrak{u}}-2 \zeta \epsilon_{x}\right) u_{x x} \\
& -2 \mu_{x} u_{x t}-3 \epsilon_{u} u_{x} u_{x x}-\mu_{\mathfrak{u}} u_{t} u_{x x}-2 \mu_{\mathfrak{u}} u_{x} u_{x t}
\end{aligned}
$$

now we choose the highest-order derivative terms in (4.3) have a factor $u_{x t}$

$$
-2 \mu_{x} u_{x t}-2 \mu_{u} u_{x} u_{x t}=0,
$$

this leads to

$$
\mu_{x}=\mu_{\mathfrak{u}}=0 .
$$

Now we replace $u_{x x}$ by the left-hand side of Eq. (4.1) and removes many terms from Eq. (4.3) containing $\mu_{\mathrm{x}}$ and $\mu_{\mathrm{t}}$. So the remaining terms are

$$
\begin{aligned}
& \zeta_{t}-\epsilon_{t} u_{x}+\left(\zeta_{\mathfrak{u}}-\mu_{t}\right) u_{t}-\zeta_{\mathfrak{u}} u_{x} u_{t}+u\left(\zeta_{x}+\left(\zeta_{\mathfrak{u}}-\epsilon_{x}\right) u_{x}-\epsilon_{\mathfrak{u}} u_{x}^{2}\right)+u_{x} \zeta \\
& \quad=\zeta_{x x}+\left(2 \zeta_{x u}-\epsilon_{x x}\right) u_{x}+\left(\zeta_{\mathfrak{u u}}-2 \epsilon_{x u}\right) u_{x}^{2}-\epsilon_{\mathfrak{u} u} u_{x}^{3}+\left(\zeta_{\mathfrak{u}}-2 \epsilon_{x}-3 \epsilon_{\mathfrak{u}} u_{x}\right)\left(u_{t}+u u_{x}\right),
\end{aligned}
$$


we choose the terms multiplied by $\mathfrak{u}_{t}$, then we get

$$
\left(\zeta_{\mathrm{u}}-\mu_{\mathrm{t}}\right) \mathrm{u}_{\mathrm{t}}-\epsilon_{\mathrm{u}} u_{x} u_{\mathrm{t}}=\left(\zeta_{\mathrm{u}}-2 \epsilon_{\mathrm{x}}-3 \epsilon_{\mathrm{u}} u_{x}\right) u_{\mathrm{t}}
$$

so we determine equations:

$$
\zeta_{\mathfrak{u}}-\mu_{\mathfrak{t}}=0, \quad \epsilon_{\mathfrak{u}}=0, \quad \zeta_{\mathfrak{u}}-2 \epsilon_{x}-3 \epsilon_{\mathfrak{u}} u_{x}=0 .
$$

We solve Eq. (4.5), this leads to

$$
\epsilon=\frac{1}{2} \mu_{t} x+\alpha(t)
$$

for some arbitrary function $\alpha$. From equation (4.4) we obtain

$$
\zeta_{\mathrm{uu}}-2 \epsilon_{\mathrm{xu}}=0, \quad \epsilon_{\mathrm{uu}}=0,
$$

since

$$
\epsilon_{x u}=0,
$$

then

$$
\zeta_{\mathrm{uu}}=0,
$$

by integrating both sides of Eq. (4.6) we obtain

$$
\zeta=\beta(x, t) u+\gamma(x, t),
$$

where $\beta$ and $\gamma$ arbitrary function. From Eq. (4.5) we obtain

$$
\mu=\beta(x, t) t+\delta(x),
$$

such that $\delta$ is arbitrary function. by determining $\alpha, \beta, \gamma$ and $\delta$ we eventually arrive at the general solution

$$
\epsilon=\beta_{1}+t \beta_{2}+x \beta_{4}+x t \beta_{5}, \quad \mu=\beta_{3}+2 t \beta_{4}+t^{2} \beta_{5}, \quad \zeta=\beta_{2}-u \beta_{4}+\left(x-u_{t}\right) \beta_{5},
$$

where $\beta_{1}, \beta_{2}, \beta_{3}, \beta_{4}$, and $\beta_{5}$ are arbitrary constants. Now we determine the symmetry of the Eq. (4.1). Since the generator

$$
X=\epsilon \frac{\partial}{\partial x}+\mu \frac{\partial}{\partial t}+\zeta \frac{\partial}{\partial u}
$$

substituting the infinitesimal (4.7) into Eq. (4.8) we get

$$
X=\beta_{1} \frac{\partial}{\partial x}+t \beta_{2} \frac{\partial}{\partial x}+x \beta_{4} \frac{\partial}{\partial x}+x t \beta_{5} \frac{\partial}{\partial x}+\beta_{3} \frac{\partial}{\partial t}+2 t \beta_{4} \frac{\partial}{\partial t}+t^{2} \beta_{5} \frac{\partial}{\partial t}+\beta_{2} \frac{\partial}{\partial u}-u \beta_{4} \frac{\partial}{\partial u}+(x-u t) \beta_{5} \frac{\partial}{\partial u},
$$

then for symmetries are given by

$$
x_{1}=\frac{\partial}{\partial x}, \quad x_{2}=\frac{\partial}{\partial t}, \quad x_{3}=t \frac{\partial}{\partial x}+\frac{\partial}{\partial u}, \quad x_{4}=x \frac{\partial}{\partial x}+2 t \frac{\partial}{\partial t}-u \frac{\partial}{\partial u}, \quad x_{5}=x t \frac{\partial}{\partial x}+t^{2} \frac{\partial}{\partial t}+(x-u t) \frac{\partial}{\partial u} .
$$

\section{Heat equation}

The heat equation is a partial differential equation that describes how the distribution of some quantity (such as heat) evolves over time in a solid medium, as it spontaneously flows from places where it is higher towards places where it is lower. It is a special case of the diffusion equation. Using a Lie symmetry group generator and a generalized for solving second order ordinary differential equations, we determine new symmetries for two dimensional heat equations, leading to new solutions. 
5.1. One-dimensional heat equation [1]

The one dimension heat equation is given by

$$
u_{x x}-u_{t}=0 .
$$

Now we determine the symmetries of the one-dimensional heat equation from invariance condition

$$
\left.X^{[2]} f\right|_{f=0}=0,
$$

by using the extended operator

$$
\chi^{[2]}=\epsilon \partial_{x}+\mu \partial_{t}+\zeta \partial_{\mathfrak{u}}+\zeta^{(x)} \partial_{u_{x}}+\zeta^{(t)} \partial_{u_{t}}+\zeta^{(x x)} \partial_{u_{x x}}+\zeta^{(x t)} \partial_{u_{x t}}+\zeta^{t t} \partial_{u_{t t}},
$$

and substituting

$$
f: u_{x x}=u_{t}
$$

we get

$$
\zeta^{(\mathrm{t})}-\zeta^{(\mathrm{xx})}=0,
$$

now substituting the extended transformations equation (2.8) and (2.10) into equation (5.1) we get

$$
\begin{aligned}
\zeta_{t} & -\epsilon_{t} u_{x}+\left(\zeta_{\mathfrak{u}}-\mu_{t}\right) u_{t}-\epsilon_{\mathfrak{u}} u_{x} u_{t}-\mu_{\mathfrak{u}} u_{t}^{2}-\zeta_{x x}-\left(2 \zeta_{x u}+\epsilon_{x x}\right) u_{x}+\mu_{x x} u_{t}-\left(\zeta_{u u}+2 \epsilon_{x u}\right) u_{x}^{2} \\
& +2 \mu_{x u} u_{x} u_{t}+\epsilon_{\mathfrak{u u}} u_{x}^{3}+\mu_{u u} u_{x}^{2} u_{t}-\left(\zeta_{\mathfrak{u}}+2 \varepsilon_{x}\right) u_{x x}+2 \mu_{x} u_{x t}+3 \epsilon_{\mathfrak{u}} u_{x} u_{x x}+\mu_{\mathfrak{u}} u_{t} u_{x x}+2 \mu_{\mathfrak{u}} u_{x} u_{x t}=0
\end{aligned}
$$

By using the heat equation to replace $u_{x x}$ by $u_{t}$ and isolating coefficients involving each power of $u_{x}$ and $u_{t}, u_{x t}$, we are left with the system of determining equations

$$
\begin{aligned}
& C: \zeta_{t}-\zeta_{x x}=0, \quad u_{x}:-\mu_{t}-2 \zeta_{x u}+\mu_{x x}=0 \text {, } \\
& u_{x}^{2}:-\zeta_{u u}-2 \mu_{x u}=0, \\
& u_{x}^{3}: \mu_{u u}=0 \text {, } \\
& u_{t} u_{x}: 2 \mu_{\mathfrak{u}}+2 \epsilon_{x u}=0, \\
& u_{t x}: 2 \epsilon_{x}=0 \text {, } \\
& \epsilon_{\mathfrak{u}}=0 \text {, } \\
& \begin{array}{r}
u_{t}:-\epsilon_{t}+\epsilon_{x x}+2 \mu_{x}=0, \\
u_{x} u_{t x}: 2 \epsilon_{\mathfrak{u}}=0, \\
\mu_{\mathfrak{u}}=0, \quad \zeta_{\mathfrak{u u}}=0 .
\end{array}
\end{aligned}
$$

Since

$$
-\epsilon_{\mathrm{t}}+\epsilon_{\mathrm{xx}}+2 \mu_{\mathrm{x}}=0
$$

then

$$
2 \mu_{\mathrm{x}}-\epsilon_{\mathrm{t}}=0,
$$

and equations

$$
\begin{aligned}
-\mu_{t}-2 \zeta_{x u}+\mu_{x x} & =0, \\
\zeta_{t}-\zeta_{x x} & =0 .
\end{aligned}
$$

From equation (5.2) we get

$$
\mu=\frac{1}{2} \epsilon_{t} x+\alpha(t) m
$$

such that $\alpha$ is an arbitrary function of $t$. Since

$$
\zeta_{\mathrm{uu}}=0,
$$

then by integration equation (5.6) twice with respect to $u$ we get

$$
\zeta=\beta(x, t) u+\gamma(x, t),
$$


where $\beta$ and $\delta$ are arbitrary functions of both $x$, t. From equations (5.2), (5.5), and (5.7) we get

$$
\mu_{x x}=0, \quad \mu_{t}=\frac{1}{2} \epsilon_{t t} x+\alpha_{t}(t), \quad \zeta_{x u}=\beta_{x}(x, t),
$$

substituting equation (5.8) into equation (5.3) we get

$$
-2 \beta_{x}(x, t)-\frac{1}{2} \epsilon_{x \chi} x-\alpha_{t}(t)=0, \quad-2 \beta_{x}(x, t)=\frac{1}{2} \epsilon_{x \chi} x+\alpha_{t}(t),
$$

then

$$
\beta_{\chi}(x, t)=\frac{-1}{4} \epsilon_{x \chi} x-\frac{1}{2} \alpha_{t}(t),
$$

we integrate both sides with respect to $x$, we get

$$
\beta(x, t)=\frac{-1}{8} \epsilon_{t t} x^{2}-\frac{1}{2} \alpha_{t}(t) x+\delta(t),
$$

where $\delta(t)$ is an arbitrary function of $t$. Now substituting equation (5.9) into equation (5.7) yields

$$
\zeta=\left(\frac{-1}{8} \epsilon_{\mathrm{tt}} x^{2}-\frac{1}{2} \alpha_{\mathrm{t}}(\mathrm{t}) \mathrm{x}+\delta(\mathrm{t})\right) \mathrm{u}+\gamma(\mathrm{x}, \mathrm{t})
$$

then from equation (5.10) we get

$$
\begin{aligned}
\zeta_{x x} & =\frac{-1}{4} \epsilon_{t t} u+\gamma_{x x}(x, t), \\
\zeta_{t} & =\left(-\frac{1}{8} \epsilon_{t t t} x^{2}-\frac{1}{2} \alpha_{t t}(t) x+\delta_{t}(t)\right) u+\gamma_{t}(x, t),
\end{aligned}
$$

substituting the equations (5.11) and (5.12) into equation (5.4) we get

$$
\left(-\frac{1}{8} \epsilon_{\mathrm{ttt}} x^{2}-\frac{1}{2} \alpha_{\mathrm{tt}}(\mathrm{t}) \mathrm{x}+\delta_{\mathrm{t}}(\mathrm{t})\right) \mathrm{u}+\gamma_{\mathrm{t}}(\mathrm{x}, \mathrm{t})+\frac{1}{4} \epsilon_{\mathrm{tt}} \mathrm{u}-\gamma_{\mathrm{xx}}(\mathrm{x}, \mathrm{t})=0,
$$

splitting equation (5.13) with respect to the powers of $x$ and $t$ we get

$$
\begin{aligned}
\epsilon_{\mathrm{ttt}} & =0, \\
\alpha_{\mathrm{tt}} & =0, \\
\delta_{\mathrm{t}}(\mathrm{t})+\frac{1}{4} \epsilon_{\mathrm{tt}} & =0, \\
\gamma_{\mathrm{t}}(x, \mathrm{t})-\gamma_{x x}(x, \mathrm{t}) & =0,
\end{aligned}
$$

integrating the equations (5.14), (5.15), and (5.16) with respect to $t$ we get

$$
\begin{aligned}
\epsilon_{\mathrm{tt}} & =\mathrm{B}_{1}, \\
\epsilon & =\frac{\mathrm{B}_{1}}{2} t^{2}+\mathrm{B}_{2} t+\mathrm{B}_{3}, \\
\alpha_{\mathrm{t}}(t) & =\mathrm{B}_{4}, \\
\alpha(t) & =B_{4} t+B_{5}, \\
\delta(t) & =-\frac{1}{4} B_{1} t+B_{6},
\end{aligned}
$$

substituting equations (5.17), (5.19), (5.21) into equation (5.10) we get

$$
\zeta=\left(\frac{-1}{8} B_{1} x^{2}-\frac{1}{2} B_{4} x-\frac{1}{4} B_{1} t+B_{6}\right) u+\gamma(x, t),
$$


now put $\beta_{1}=\frac{B_{1}}{2}, \beta_{2}=\frac{B_{2}}{2}, \beta_{3}=B_{3}, \beta_{4}=B_{4}, \beta_{5}=B_{5}$ and $\beta_{6}=B_{6}$, then the equation (5.22) and (5.18) become

$$
\epsilon=\beta_{1} t^{2}+2 \beta_{2} t+\beta_{3}, \quad \zeta=\frac{-1}{4} \beta_{1} x^{2} u-\frac{\beta_{4}}{2} x u-\frac{1}{2} \beta_{1} t u+\beta_{6} u+\gamma(x, t) .
$$

From equation (5.18), we get

$$
\epsilon_{\mathrm{t}}=\mathrm{B}_{1} \mathrm{t}+\mathrm{B}_{2}
$$

substituting equations (5.23) and (5.20) into equation (5.5) we get

$$
\mu=\frac{1}{2}\left(B_{1} t+B_{2}\right) x+B_{4} t+B_{5}=\frac{B_{1}}{2} x t+\frac{B_{2}}{2} x+B_{4} t+B_{5}=\beta_{1} x t+\beta_{2} x+\beta_{4} t+\beta_{5} .
$$

In the end we arrive the infinitesimal

$$
\epsilon=\beta_{1} t^{2}+2 \beta_{2} t+\beta_{3}, \quad \mu=\beta_{1} x t+\beta_{2} x+\beta_{4} t+\beta_{5}, \quad \zeta=\frac{-1}{4} \beta_{1} x^{2} u-\frac{\beta_{4}}{2} x u-\frac{1}{2} \beta_{1} t u+\beta_{6} u+\gamma(x, t) .
$$

Since the generator

$$
X=\epsilon \frac{\partial}{\partial x}+\mu \frac{\partial}{\partial t}+\zeta \frac{\partial}{\partial u}
$$

substituting the infinitesimal in equation (5.24) we get

$$
\begin{array}{r}
X=\beta_{1} t^{2} \frac{\partial}{\partial x}+2 \beta_{2} t \frac{\partial}{\partial x}+\beta_{3} \frac{\partial}{\partial x}+\beta_{1} x t \frac{\partial}{\partial t}+\beta_{2} x \frac{\partial}{\partial t}+\beta_{4} t \frac{\partial}{\partial t}+\beta_{5} \frac{\partial}{\partial t} \\
-\frac{1}{4} \beta_{1} x^{2} u \frac{\partial}{\partial u}-\frac{\beta_{4}}{2} x u \frac{\partial}{\partial u}-\frac{1}{2} \beta_{1} t u \frac{\partial}{\partial u}+\beta_{6} u \frac{\partial}{\partial u}+\gamma(x, t) \frac{\partial}{\partial u},
\end{array}
$$

therefore the symmetries are given by

$$
\begin{aligned}
& X_{1}=\frac{\partial}{\partial x}, \\
& x_{2}=\frac{\partial}{\partial t}, \\
& X_{3}=x t \frac{\partial}{\partial t}+t^{2} \frac{\partial}{\partial x}-\left(\frac{1}{4} x^{2}+\frac{1}{2} u\right) u \frac{\partial}{\partial u}, \\
& x_{4}=x \frac{\partial}{\partial t}+2 t \frac{\partial}{\partial x}, \\
& X_{5}=u \frac{\partial}{\partial u}, \\
& x_{6}=t \frac{\partial}{\partial t}-\frac{1}{2} x u \frac{\partial}{\partial u}, \quad x_{\infty}=\gamma(x, t) \frac{\partial}{\partial u} \text {. }
\end{aligned}
$$

\subsection{Two-dimensional heat equation [19]}

We use a Lie symmetry group generator and a generalized form of Manale's formula for solving second order ordinary differential equations, we determine new symmetries for the two dimensional heat equations. The two dimension heat equation is given

$$
u_{t}-u_{x x}-u_{y y}=0,
$$

in which $u$ is dependent variable and $t, x$ are independent variables. The infinitesimal generator is

$$
x=\epsilon(x, y, t, u) \frac{\partial}{\partial x}+\varphi(x, y, t, u) \frac{\partial}{\partial y}+\mu(x, y, t, u) \frac{\partial}{\partial t}+\zeta(x, y, t, u) \frac{\partial}{\partial u},
$$

where $X^{[2]}$ is the second extended operator of $X$ given by

$$
\begin{aligned}
X^{[2]}= & X+\zeta^{(x)} \frac{\partial}{\partial u_{x}}+\zeta^{(y)} \frac{\partial}{\partial u_{y}}+\zeta^{(t)} \frac{\partial}{\partial u_{t}}+\zeta^{(x x)} \frac{\partial}{\partial u_{x x}}+\zeta^{(y y)} \frac{\partial}{\partial u_{y y}} \\
& +\zeta^{(t t)} \frac{\partial}{\partial u_{t t}}+\zeta^{(x y)} \frac{\partial}{\partial u_{x y}}+\zeta^{(x t)} \frac{\partial}{\partial u_{x t}}+\zeta^{(y t)} \frac{\partial}{\partial u_{y t}}
\end{aligned}
$$

with the symmetry condition

$$
\left.X^{[2]}\left(u_{t}-u_{x x}-u_{y y}\right)\right|_{\left(u_{y y}=u_{t}-u_{x x}\right)}=0
$$


then we get

$$
\zeta^{(\mathfrak{t})}-\zeta^{(x x)}-\left.\zeta^{(y y)}\right|_{\left(\mathfrak{u}_{y y}=\mathfrak{u}_{\mathfrak{t}}-\mathfrak{u}_{x x}\right)}=0,
$$

where

$$
\begin{aligned}
\zeta= & f u+g \\
\zeta^{(x)}= & D_{x}(\zeta)-u_{x} D_{x}(\epsilon)-u_{t} D_{x}(\mu)-u_{y} D_{x}(\varphi) \\
= & D_{x}(f u+g)-u_{x} D_{x}(\epsilon)-u_{t} D_{x}(\mu)-u_{y} D_{x}(\varphi) \\
= & g_{x}+u f_{x}+u_{x}\left(f-\epsilon_{x}\right)-u_{t} \mu_{x}-u_{y} \varphi_{x} \\
\zeta^{(x x)}= & D_{x}\left(\zeta^{(x)}\right)-u_{x x} D_{x}(\epsilon)-u_{x t} D_{x}(\mu)-u_{x y} D_{x}(\varphi) \\
= & D_{x}\left(g_{x}+u f_{x}+u_{x}\left(f-\epsilon_{x}\right)-u_{t} \mu_{x}-u_{y} \varphi_{x}\right)-u_{x x} \epsilon_{x}-u_{x t} \mu_{x}-u_{x y} \varphi_{x} \\
= & g_{x x}+u_{x} f_{x}+u f_{x x}+u_{x} f_{x}+u_{x x} f-u_{x} \epsilon_{x x}-u_{x x} \epsilon_{x}-u_{x t} \mu_{x}-u_{t} \mu_{x x} \\
& -u_{y} \varphi_{x x}-u_{x y} \varphi_{x}-u_{x x} \epsilon_{x}-u_{x t} \mu_{x}-u_{x y} \varphi_{x} \\
= & g_{x x}+u f_{x x}+u_{x}\left[2 f_{x}-\epsilon_{x x}\right]-u_{y} \varphi_{x x}-u_{t} \mu_{x x}+u_{x x}\left[f-2 \epsilon_{x}\right]-2 u_{x y} \varphi_{x}-2 u_{x t} \mu_{x}, \\
\zeta^{(t)}= & D_{t}(\zeta)-u_{t} D_{t}(\epsilon)-u_{y} D_{t}(\varphi)-u_{t} D_{t}(\mu) \\
= & D_{t}(f u+g)-u_{x} D_{t}(\epsilon)-u_{y} D_{t}(\varphi)-u_{t} D_{t}(\mu) \\
= & u_{t} f+u f_{t}+g_{t}-u_{x} \epsilon_{t}-u_{y} \varphi_{t}-u_{t} \mu_{t}=g_{t}+u f_{t}+u_{t}\left[f-\mu_{t}\right]-\epsilon_{t} u_{x}-u_{y} \varphi_{t}, \\
\zeta^{(y)}= & D_{y}(\zeta)-u_{x} D_{y}(\epsilon)-u_{y} D_{y}(\varphi)-u_{t} D_{y}(\mu)=g_{y}+u f_{y}+u_{y}\left[f-\varphi_{y}\right]-u_{x} \epsilon_{y}-u_{t} \mu_{y}, \\
\zeta^{(y y)}= & D_{y}\left(\zeta^{y}\right)-u_{x y} D_{y}(\epsilon)-u_{y y} D_{y}(\varphi)-u_{y t} D_{y}(\mu) \\
= & g_{y y}+u f_{y y}+u_{y} f_{y}+u_{y} f_{y}+u_{y y} f-u_{y} \varphi_{y y}-u_{y y} \varphi_{y} \\
& -u_{x} \epsilon_{y y}-u_{x y} \epsilon_{y}-u_{t} \mu_{y y}-u_{y t} \mu_{y}-u_{x y} \epsilon_{y}-u_{y y} \varphi_{y}-u_{y t} \mu_{y} \\
= & g_{y y}+u f_{y y}+u_{y}\left[2 f_{y}-\varphi_{y y}\right]-u_{x} \epsilon_{y y}-u_{t} \mu_{y y}+u_{y y}\left[f-2 \varphi_{y}\right]-2 u_{x y} \epsilon_{y}-2 u_{y t} \mu_{y} .
\end{aligned}
$$

Now substituting Eqs. (5.29), (5.28), (5.30) in Eq. (5.26) and using heat equation to replace $u_{y y}$ by $u_{t}-u_{x x}$ yields

$$
\begin{aligned}
g_{t}+ & u f_{t}+u_{t}\left[f-\mu_{t}\right]-\epsilon_{t} u_{x}-u_{y} \varphi_{t} \\
= & g_{x x}+u f_{x x}+u_{x}\left[2 f_{x}-\epsilon_{x x}\right]-u_{y} \varphi_{x x}-u_{t} \mu_{x x}+u_{x x}\left[f-2 \epsilon_{x}\right]-2 u_{x y} \varphi_{x}-2 u_{x t} \mu_{x} \\
& +g_{y y}+u f_{y y}+u_{y}\left[2 f_{y}-\varphi_{y y}\right]-u_{x} \epsilon_{y y}-u_{t} \mu_{y y}+\left(u_{t}-u_{x x}\right)\left[f-2 \varphi_{y}\right]-2 u_{x y} \epsilon_{y}-2 u_{y t} \mu_{y}
\end{aligned}
$$

more simplifying the equation above yields

$$
\begin{aligned}
& \left(g_{t}-g_{x x}-g_{y y}\right)+u\left(f_{t}-f_{x x}-f_{y y}\right)+u_{t}\left(-\mu_{t}+\mu_{x x}+\mu_{y y}+2 \varphi_{y}\right) \\
& \quad+u_{x}\left(-\epsilon_{t}+\epsilon_{x x}+\epsilon_{y y}+2 f_{x}\right)+u_{y}\left(\varphi_{y y}-\varphi_{t}+\varphi_{x x}-2 f_{y}\right) \\
& \quad+u_{x x}\left(2 \epsilon_{x}-2 \varphi_{y}\right)+u_{x y}\left(2 \varphi_{x}+2 \epsilon_{y}\right)+2 u_{x t} \mu_{x}+2 u_{y t} \mu_{y}=0
\end{aligned}
$$

separating coefficients in Eq. (5.30) we get

$$
\begin{aligned}
C: g_{t}-g_{x x}-g_{y y} & =0, \\
u: f_{t}-f_{x x}-f_{y y} & =0, \\
u_{t}: \mu_{t}-\mu_{x x}-\mu_{y y}-2 \varphi_{y} & =0, \\
u_{x}: \epsilon_{t}-\epsilon_{x x}-\epsilon_{y y}+2 f_{x} & =0, \\
u_{y}: \varphi_{t}-\varphi_{x x}-\varphi_{y y}+2 f_{y} & =0, \\
u_{x x}: \epsilon_{x}-\varphi_{y} & =0, \\
u_{x y}: \varphi_{x}+\epsilon_{y} & =0, \\
u_{x t}: \mu_{x} & =0,
\end{aligned}
$$




$$
u_{y t}: \mu_{y}=0
$$

integrating Eq. (5.37) with respect to $x$ we get

$$
\mu=\alpha(t),
$$

since $\mu_{x x}=0$ and $\mu_{y y}=0$, then from Eq. (5.32) we get

$$
\varphi=\frac{1}{2} \alpha_{\mathrm{t}} \mathrm{y}+\beta(x, \mathrm{t}),
$$

differentiating Eq. (5.38) with respect to $x$ and $y$ twice yields

$$
\varphi_{x x}=\beta_{x x}(x, t), \quad \varphi_{y y}=0 .
$$

Differentiating Eq. (5.38) with respect to $t$ and $y$ we get

$$
\varphi_{\mathrm{tt}}=\frac{1}{2} \alpha_{\mathrm{tt}} \mathrm{y}+\beta_{\mathrm{t}}(\mathrm{x}, \mathrm{t}), \quad \varphi_{\mathrm{y}}=\frac{1}{2} \alpha_{\mathrm{t}} .
$$

Substituting $\varphi_{y}$ into Eq. (5.35) yields

$$
\epsilon_{\mathrm{x}}=\frac{1}{2} \alpha_{\mathrm{t}}
$$

integrating Eq. (5.39) we get

$$
\epsilon=\frac{1}{2} \alpha_{t} x+\gamma(y, t)
$$

differentiating Eq. (5.40) with respect to $t$ we get

$$
\epsilon_{\mathrm{t}}=\frac{1}{2} \alpha_{\mathrm{t} t} x+\gamma_{\mathrm{t}}(\mathrm{y}, \mathrm{t})
$$

Differentiating Eq. (5.40) with respect to $x$ and $y$ twice yields

$$
\epsilon_{x x}=0, \quad \epsilon_{y y}=\gamma_{y y}(y, t) .
$$

Differentiating Eq. (5.36) with respect to $x$ and $y$, respectively we get

$$
\varphi_{x x}=0=\beta_{x x}(x, t), \quad \epsilon_{y y}=0=\gamma_{y y}(y, t),
$$

integrating Eq. (5.41) twice yields

$$
\beta(x, t)=B_{1} x+B_{2}, \quad \gamma(y, t)=B_{3} y+B_{4} .
$$

Substituting $\epsilon_{t}, \epsilon_{x x}, \epsilon_{y y}$ into Eq. (5.33) we obtain

$$
\frac{1}{2} \alpha_{t t}(t) x+\gamma_{t}(y, t)+2 f_{x}=0,
$$

then

$$
f_{x}=\frac{-1}{4} \alpha_{t t} x-\frac{1}{2} \gamma_{t}(y, t),
$$

integrating Eq. (5.42) with respect to $x$ yields

$$
f=\frac{-1}{8} \alpha_{t t} x^{2}-\frac{1}{2} \gamma_{t}(y, t) x+\delta(y, t) .
$$


Substituting $\epsilon_{\mathrm{t}}, \epsilon_{x x}, \epsilon_{y y}$ into Eq. (5.34) we obtain

$$
f=\frac{-1}{8} \alpha_{t t} y^{2}-\frac{1}{2} \beta_{t}(x, t) y+\kappa(x, t) .
$$

From Eqs. (5.43) and (5.42) we obtain

$$
f=-\frac{1}{8} \alpha_{t t} x^{2}-\frac{1}{8} \alpha_{t t} y^{2}-\frac{1}{2} \gamma_{t}(y, t)-\frac{1}{2} \beta_{t}(x, t) y+\kappa(x, t)+\delta(y, t) .
$$

Differentiating Eq. (5.43) with respect to $t$ and twice with respect to $x$ and $y$ we get

$$
\begin{aligned}
f_{t} & =-\frac{1}{8} \alpha_{t t t} x^{2}-\frac{1}{8} \alpha_{t t t} y^{2}-\frac{1}{2} \gamma_{t} t(y, t)-\frac{1}{2} \beta_{t} t(x, t) y+\kappa_{t}(x, t)+\delta_{t}(y, t), \\
f_{x x} & =-\frac{1}{4} \alpha_{t t}+\kappa_{x x}(x, t), \quad f_{y y}=-\frac{1}{2} \alpha_{t t}+\delta_{y y}(y, t),
\end{aligned}
$$

substituting Eqs. (5.45) and (5.46) into Eq. (5.31) yields

$$
-\frac{1}{8} \alpha_{t t t} x^{2}-\frac{1}{8} \alpha_{t t t} y^{2}-\frac{1}{2} \gamma_{t} t(y, t)-\frac{1}{2} \beta_{t} t(x, t) y+\kappa_{t}(x, t)+\delta_{t}(y, t)+\frac{1}{2} \alpha_{t t}-\delta_{y y}(y, t)-\kappa_{x x}(x, t)=0 .
$$

Splitting Eq. (5.47) we get

$$
\begin{aligned}
\alpha_{\mathrm{ttt}}(\mathrm{t}) & =0, \\
\kappa_{x x}(x, t) & =0, \\
\delta_{y y}(y, t) & =0, \\
\kappa_{t}(x, t) & =0, \\
\delta_{t}(y, t) & =0,
\end{aligned}
$$

integrating with respect to $t, x, y$ in Eqs. (5.48), (5.49), (5.50), respectively yields

$$
\alpha(t)=\frac{1}{2} B_{5} t^{2}+B_{6} t+B_{7}, \quad k(x, t)=B_{8} x+B_{9}, \quad \delta(y, t)=B_{10} y+B_{11} .
$$

Since

$$
\mu=\alpha(t)
$$

then

$$
\mu=\frac{1}{2} B_{5} t^{2}+B_{6} t+B_{7}
$$

Substituting $\alpha_{t}(t)$ and $\gamma(y, t)$ into Eq. (5.40) we obtain

$$
\epsilon=\frac{1}{2} B_{5} x t+\frac{1}{2} B_{6} x+B_{3} y+B_{4} .
$$

Substituting $\alpha_{t}(t)$ and $\beta(x, t)$ in Eq. (5.38) we get

$$
\varphi=\frac{1}{2} B_{5} y t+\frac{1}{2} B_{6} y+B_{1} x+B_{2}
$$

Since

$$
\alpha_{t t}=B_{5}, \quad \gamma_{t}(y, t)=0, \quad \beta_{t}(x, t)=0, \quad \kappa_{t}(x, t)=B_{8} x+B_{9}, \quad \delta_{t}(y, t)=B_{10} y+B_{11},
$$

then the Eq. (5.44) becomes

$$
f=-\frac{1}{8} B_{5} x^{2}-\frac{1}{8} B_{5} y^{2}+B_{8} x+B_{9}+B_{10} y+B_{11}
$$


Now we put $\beta_{1}=\frac{1}{2} B_{5}, \beta_{2}=\frac{1}{2} B_{6}, \beta_{3}=B_{7}, \beta_{4}=B_{3}, \beta_{5}=B_{4}, \beta_{6}=B_{1}, \beta_{7}=B_{2}, \beta_{8}=B_{8}, \beta_{9}=B_{10}$, $\beta_{10}=B_{9}+B_{11}$. Then we get infinitesimals:

$$
\begin{array}{ll}
\mu=\beta_{1} t^{2}+2 \beta_{2} t+\beta_{3}, & \epsilon=\beta_{1} x t+\beta_{2} x+\beta_{4} y+\beta_{5}, \\
\varphi=\beta_{1} y t+\beta_{2} y+\beta_{6} x+\beta_{7}, & f=-\frac{1}{4} \beta_{1}\left(x^{2}+y^{2}\right)+\beta_{8} x+\beta_{9} y+\beta_{10} .
\end{array}
$$

From Eq. (5.27), then $\zeta$ becomes

$$
\zeta=-\frac{1}{4} u \beta_{1}\left(x^{2}+y^{2}\right)+\beta_{8} x u+\beta_{9} y u+\beta_{10} u+g .
$$

Since the generator $X$ in Eq. (5.25) is

$$
X=\epsilon(x, y, t, u) \frac{\partial}{\partial x}+\varphi(x, y, t, u) \frac{\partial}{\partial y}+\mu(x, y, t, u) \frac{\partial}{\partial t}+\zeta(x, y, t, u) \frac{\partial}{\partial u^{\prime}}
$$

substituting infinitesimals Eq. (5.51) in generator $X$ yields

$$
\begin{gathered}
x=\left(t^{2} \frac{\partial}{\partial t}+x t \frac{\partial}{\partial x}+t y \frac{\partial}{\partial y}-\frac{1}{4} u\left(x^{2}+y^{2}\right) \frac{\partial}{\partial u}\right) \beta_{1}+\left(2 t \frac{\partial}{\partial t}+x \frac{\partial}{\partial x}+y \frac{\partial}{\partial y}\right) \beta_{2}+\frac{\partial}{\partial t} \beta_{3} \\
+\beta_{4} y \frac{\partial}{\partial x}+\beta_{5} \frac{\partial}{\partial x}+x \beta_{6} \frac{\partial}{\partial y}+\beta_{7} \frac{\partial}{\partial y}+\beta_{8} x u \frac{\partial}{\partial u}+\beta_{9} y u \frac{\partial}{\partial u}+\beta_{10} u \frac{\partial}{\partial u}+g \frac{\partial}{\partial y}
\end{gathered}
$$

therefore the symmetries are given by

$$
\begin{aligned}
& X_{1}=\frac{\partial}{\partial t}, \\
& x_{2}=\frac{\partial}{\partial x}, \quad x_{3}=y \frac{\partial}{\partial x}, \\
& x_{4}=x \frac{\partial}{\partial y} \\
& x_{5}=\frac{\partial}{\partial y}, \quad x_{6}=x u \frac{\partial}{\partial u}, \\
& x_{7}=y u \frac{\partial}{\partial u}, \\
& X_{10}=2 t \frac{\partial}{\partial t}+x \frac{\partial}{\partial x}+y \frac{\partial}{\partial y}, \quad x_{\infty}=g \frac{\partial}{\partial y} \text {. } \\
& x_{9}=t^{2} \frac{\partial}{\partial t}+t x \frac{\partial}{\partial x}+t y \frac{\partial}{\partial y}-\frac{1}{4} u\left(x^{2}+y^{2}\right) \frac{\partial}{\partial u},
\end{aligned}
$$

\section{References}

[1] C. M. Adams, A Lie Symmetry analysis of the heat equation through modified one-parameter local point transformation, Doctoral Dissertation (University of South Africa), South Africa, (2014). 5.1

[2] C. Andreas, Lie Algebras and Representation Theory, Universitat Wien, Nordbergstr, (2009).

[3] D. J. Arrigo, Symmetry Analysis of Differential Equations, John Wiley \& Sons, Hoboken, (2015).

[4] M. Azadi, H. Jafari, Lie Symmetry Reductions of a Coupled KdV System of Fractional Order, Nonlinear Dyn. Syst. Theory, 18 (2018), 22-28. 1

[5] D. Baleanu, H. K. Jassim, A novel approach for Korteweg-de Vries equation of fractional order, J. Appl. Comput. Mech., 5 (2019), 192-198.

[6] D. Baleanu, H. K. Jassim, H. Khan, A Modification Fractional Variational Iteration Method for solving Nonlinear Gas Dynamic and Coupled KdV Equations Involving Local Fractional Operators, Thermal Sci., 22 (2018), 165-175. 1

[7] H. Bateman, Some recent researches on the motion of fluids, Monthly Weather Review, 43 (1915), 163-170. 4

[8] G. W. Bluman, S. C. Anco, Symmetry and Integration Methods for Differential Equations, Springer, New York (2002). 1

[9] G. W. Bluman, S. Kumei, Symmetries and differential equations, Springer-Verlag, New York, (1989). 1, 2.4, 2.7

[10] J. M. Burgers, A mathematical model illustrating the theory of turbulence, Academic Press, New York, (1948). 4

[11] M. Humi, W. Miller, Second Course in Ordinary Differential Equations for Scientists and Engineers, Springer-Verlag, New York, (1988). 2.6

[12] P. E. Hydon, Hydon symmetry methods for differential equation: a beginners guide, Cambridge university press, Cambridge, (2000). 1, 2.1, 2.2, 3

[13] N. H. Ibragimov, Elementary Lie group analysis and ordinary differential equations, John Wiley \& Sons, Chichester, (1999). 1, 2.8 
[14] H. Jafari, K. H. Jassim, J. Vahidi, Reduced Differential Transform and Variational Iteration Methods for 3D Diffusion Model in Fractal Heat Transfer within Local Fractional Operators, Thermal Sci., 22 (2018), 301-307. 1

[15] H. Jafari, N. Kadkhoda, D. Baleanu, Fractional Lie group method of the time-fractional Boussinesq equation, Nonlinear Dynam., 81 (2015), 1569-1574.

[16] H. Jafari, N. Kadkhoda, C. M. Khalique, Application of Lie Symmetry Analysis and Simplest Equation Method for Finding Exact Solutions of Boussinesq Equations, Math. Probl. Eng., 2013 (2013), 4 pages.

[17] H. K. Jassim, Analytical Approximate Solutions for Local Fractional Wave Equations, Math. Methods Appl. Sci., 43 (2020), 939-947.

[18] H. K. Jassim, W. A. Shahab, Fractional variational iteration method for solving the hyperbolic telegraph equation, J. Phys. Conf. S., 1032 (2018), 1-9. 1

[19] J. M. Manale, New Symmetries of the heat equation and application to thin plate heat conduction, In Proceedings of the 2013 International Conference on Mathematics and Computation (Venice, Italy), 2013 (2013), 90-103. 5.2

[20] L. Muhsen, The Classification of Delay Differential Equations Using Lie Symmetry Analysis, Doctoral dissertation (Universiti Teknologi Malaysia), Malaysia, (2016). 2.3, 2.5, 2.6

[21] G. B. Whitham, Linear and nonlinear waves, John Wiley \& Sons, New York, (2011). 4 